\title{
The effect of ketogenic and low carb diet (Cyclic Alternation) on the ovarian morphology and insulin metabolism in polycystic ovary syndrome patients
}

\author{
Ennio Avolio ${ }^{1,2,5 *}$, Simona Ferraro ${ }^{1,2}$, Rita Mocciaro ${ }^{4}$, Giovanni De Pergola ${ }^{7}$, Fabio Buzzanca ${ }^{2}$, Roberta Venturella ${ }^{3}$, Claudio Pecorella ${ }^{2}$, \\ Giuseppe Palma ${ }^{6}$, Lorenzo Romano ${ }^{1}$, Sushil K. Mahata ${ }^{5}$, Antonino De Lorenzo ${ }^{1}$ and Marco Marchetti ${ }^{1}$ \\ ${ }^{1}$ Department of Biomedicine and Prevention, Section of Clinical Nutrition and Nutrigenomic, University of Roma "Tor Vergata", Roma, Italy \\ ${ }^{2}$ Health Center srl, via Sabotino 56, 87100 Cosenza, Italy \\ ${ }^{3}$ Department of Experimental and Clinical Medicine, Unit of Obstetrics and Gynaecology, Reproductive Assisted Technology Centre, Magna Graecia University \\ of Catanzaro, Italy \\ ${ }^{4}$ Department of Obstetrics and Gynaecology, "SS. Annunziata” Hospital, Cosenza, Italy \\ ${ }^{5}$ Department of Medicine, University of California, San Diego, 9500 Gilman Drive, La Jolla, CA, 92093-0732, USA \\ ${ }^{6}$ S.S.D. Animal Experimentation, National Institute of Cancer IRCCS-"Fondazione G. Pascale", Naples, Italy \\ ${ }^{7}$ Clinical Nutrition Unit, Medical Oncology, Department of Biomedical Science and Human Oncology, University of Bari, School of Medicine, 70124 Bari, Italy
}

\begin{abstract}
Objective: Test the hypothesis that the ketogenic and low carb treatment (cyclic diet) in the polycystic ovary syndrome (PCOS) phenotype decreases the number of ovarian follicles and improves condition of insulin resistance in women with amenorrhea.

Design: Observational study.

Setting: University-affiliated hospitals.

Patient(s): Sixty PCOS women aged 18-38 years with BMI 21-45 and amenorrhea, according to 3 Rotterdam criteria 2003 (at least 2 on 3), have been enrolled; only 3 of them have not adhered to the proposed diet and have been excluded from the study.

Intervention(s): Women have been admitted to cyclic alternation of ketogenic and low carb diet (cyclic diet) for 6 months. During the diet regimen, nutritional examination, gynecological ultrasound, menstrual cycle characteristics evaluation, and blood assays were performed by means of standardized protocols.

Main Outcome Measure(s): Unadjusted and adjusted odds ratios were calculated for the effect of cyclic diet on anthropometric measurements with bioimpedance and BMI calculation, number of ovarian follicles, blood parameters such as blood count, blood glucose, insulin, and HOMA index.

Result(s): Our analysis demonstrated significant decrease in fat mass amount, HOMA index, and number of ovarian follicles after 6 months of cyclic diet administration on PCOS women with amenorrhea. These effect have not been demonstrated at 3 months interim-evaluation. In addition, after 6 months of cyclic diet, significant proportion of women experienced menstrual cycle recovery.

Conclusion(s): The Cycle administration of Ketogenic and Low Carb diet for 6 months in women with PCOS phenotype and amenorrhea seems to improve insulin resistance of these patients along with significant improvement in their ovarian morphology and menstrual cycle cyclicity.
\end{abstract}

\section{Introduction}

Polycystic ovary syndrome (PCOS) is a major contributor to subfertility, diabetes and cardiovascular disease in women [1]. The disorder is heterogeneous, encompassing a broad spectrum of signs and symptoms of ovarian dysfunction. The classic presentation, as described by Stein and Leventhal, with features of obesity, amenorrhea and hirsutism is one end of the spectrum that, at the other end, includes women with normal menstrual cyclicity and yet ultrasound evidence of a polycystic ovarian appearance [2,3]. Numerous interventions (pharmacological, non-pharmacological and surgical) have been evaluated in women with PCOS to target the reproductive, androgenic, metabolic, weight-related and psychological outcomes associated with the condition [4]. Although PCOS is the most common cause of anovulatory infertility [5], many women may remain undiagnosed in the community. However, robust conclusions have not been made due to variations in the scope, quality and result findings.

${ }^{\star}$ Correspondence to: Ennio Avolio, $\mathrm{PhD}$, Department of Biomedicine and Prevention, Section of Clinical Nutrition and Nutrigenomic, University of Rome “Tor Vergata", Rome, Italy, E-mail: ennioavolio@libero.it

Key words: cyclic diet, ketogenic, low carb, menstrual cycle, PCOS

Received: May 21, 2020; Accepted: August 20, 2020; Published: August 26, 2020 

patients

The expression of PCOS symptoms is indeed multifaceted, and the reduced conception rates associated with PCOS may be related to hyperandrogenism, obesity and insulin resistance [6]. Over the last 20 years, the body of evidence indicating that increased insulin resistance and compensatory high insulin concentrations (hyperinsulinemia) play a key role in the pathogenesis of PCOS has grown [7]. Insulin resistance is more common in overweight women but can also occur in slim women with the disorder $[8,9]$. In the last couple years, there is an increasing interest in the effect of nutritional supplements and diet therapies together with interventions on lifestyle to optimize the weight and insulin resistance of the women with PCOS [10]. Recent studies have shown that a low-carbohydrate, ketogenic diet can lead to weight loss and improvements in insulin resistance $[11,12]$ that may be beneficial for PCOS. Low carbohydrates therapy provided positive results in patients affected by obesity and type 2 diabetes, so these dietetic regimens could represent a fascinating dietetic treatment for the management of PCOS.

On the basis of these indications, it was the intention of the present work to address the effect of ketogenic and low carb cyclic diet in women affected by PCOS. Patients were excluded in cases of endocrine (except that of insulin-resistance), metabolic, gastrointestinal, hepatic, renal or cardiovascular disorders, HIV, cancer; oral, cortisone, antiinflammatory, antibiotic contraceptive therapy, pregnancy or lactation. None woman undergoing the protocol was being treated with hypoglycemic drugs such as metformin and patients were asked not to significantly change their lifestyle habits regarding physical activity (Table 1). This approach could constitute a novel therapeutic nutritional treatment in women with PCOS and amenorrhea consequently to insulin resistance.

\section{Materials and methods}

\section{Subjects}

Sixty PCOS women, ages 18-38 years, presenting at the University of Tor Vergata Rome, from January to September 2018 were included; only 3 of them have not adhered to the proposed diet and have been excluded from the study. PCOS was diagnosed according to 3 Rotterdam criteria 2003 (at least 2 on 3). Patients were excluded in cases of endocrine (except that of insulin resistance), metabolic, gastrointestinal, hepatic, renal or cardiovascular disorders, HIV, cancer; oral, cortisone, anti-inflammatory, antibiotic contraceptive therapy, pregnancy or lactation. None woman undergoing the protocol was being treated with hypoglycemic drugs such as metformin and patients were asked not to significantly change their lifestyle habits regarding physical activity. Their data were obtained prospectively during the first visit and then maintained in a computerized database (Alpha Four v. 6.0; Alpha Software). The Institutional Board at the University of Tor Vergata Rome, approved the study. The time sheet of experiments was divided in T0 (at the enrollment) before starting the diet, T1 (3 months) and T2 (6 months after the start of the dietetic regimen). The nutritional protocol was administrated at $\mathrm{T} 0$ and consisted of 21 days of ketogenic regimen followed by low carb diet for other 20 days (Figure 1). At $\mathrm{T} 0, \mathrm{~T} 1$ and $\mathrm{T} 2$ we evaluated the nutritional and gynecological parameters plus blood analysis, as detailed behind. Before the start of the study, dietitians educated each patient for at least 60 minutes on the characteristics of the diet to be followed and on the basic information concerning lifestyle and the eating cognitive behaviour. Afterwards, the patients were interviewed every 28 days by a dietitian, who checked whether patients were effectively adhering to the proposed diet. On

Table 1. Effect of cyclic diet on metabolic parameters after 3 and 6 months of treatment in 10 women

\begin{tabular}{|c|c|c|c|c|c|c|c|c|c|}
\hline ID & $\begin{array}{c}\text { Age } \\
\text { Years }\end{array}$ & $\begin{array}{c}\text { Time } \\
\text { Months } \\
\text { 0-3-6 }\end{array}$ & $\begin{array}{c}\text { Weight } \\
\text { Kg }\end{array}$ & $\begin{array}{l}\text { Height } \\
\text { cm }\end{array}$ & $\begin{array}{c}\text { BMI } \\
\text { W/H' }\end{array}$ & $\begin{array}{c}\text { Waist } \\
\text { circumference } \\
\mathrm{cm}\end{array}$ & $\begin{array}{c}\text { Fat Mass } \\
\%\end{array}$ & $\begin{array}{l}\text { Insulin } \\
\mu \mathrm{IU} / \mathrm{ml}\end{array}$ & $\begin{array}{c}\text { Glucose } \\
\text { mg/dl }\end{array}$ \\
\hline 1 & 22 & $\begin{array}{l}\mathrm{T} 0 \\
\mathrm{~T} 1 \\
\mathrm{~T} 2\end{array}$ & $\begin{array}{c}100 \\
93 \\
89\end{array}$ & 155 & $\begin{array}{l}41.6 \\
38.7 \\
37.0 \\
\end{array}$ & $\begin{array}{l}94 \\
88 \\
85\end{array}$ & $\begin{array}{l}41.6 \\
40.9 \\
36.3 \\
\end{array}$ & $\begin{array}{l}25 \\
22 \\
18\end{array}$ & $\begin{array}{c}101 \\
90 \\
85\end{array}$ \\
\hline 2 & 35 & $\begin{array}{l}\mathrm{T} 0 \\
\mathrm{~T} 1 \\
\mathrm{~T} 2\end{array}$ & $\begin{array}{c}79.5 \\
74 \\
70 \\
\end{array}$ & 150 & $\begin{array}{l}35.3 \\
32.8 \\
31.1 \\
\end{array}$ & $\begin{array}{l}91 \\
84 \\
81 \\
\end{array}$ & $\begin{array}{l}39.2 \\
38.7 \\
35.8 \\
\end{array}$ & $\begin{array}{c}26 \\
23.2 \\
20\end{array}$ & $\begin{array}{l}93 \\
88 \\
82\end{array}$ \\
\hline 3 & 25 & $\begin{array}{l}\text { T0 } \\
\text { T1 } \\
\text { T2 }\end{array}$ & $\begin{array}{l}82 \\
77 \\
74\end{array}$ & 173 & $\begin{array}{l}27.4 \\
25.7 \\
24.7\end{array}$ & $\begin{array}{l}79 \\
73 \\
71\end{array}$ & $\begin{array}{l}36.0 \\
31.0 \\
29.6\end{array}$ & $\begin{array}{c}20.8 \\
18 \\
16\end{array}$ & $\begin{array}{l}86 \\
84 \\
83\end{array}$ \\
\hline 4 & 32 & $\begin{array}{l}\text { T0 } \\
\text { T1 } \\
\text { T2 }\end{array}$ & $\begin{array}{l}65.5 \\
61.5 \\
56.5\end{array}$ & 164 & $\begin{array}{l}24.3 \\
22.8 \\
21.0\end{array}$ & $\begin{array}{c}74.5 \\
69.5 \\
67\end{array}$ & $\begin{array}{l}25.3 \\
22.2 \\
17.3\end{array}$ & $\begin{array}{c}15.6 \\
13.2 \\
11\end{array}$ & $\begin{array}{l}96 \\
93 \\
88\end{array}$ \\
\hline 5 & 38 & $\begin{array}{l}\text { T0 } \\
\text { T1 } \\
\text { T2 }\end{array}$ & $\begin{array}{c}102 \\
96 \\
94.5\end{array}$ & 153 & $\begin{array}{l}43.5 \\
41.0 \\
40.3\end{array}$ & $\begin{array}{l}115 \\
107 \\
103\end{array}$ & $\begin{array}{l}47.6 \\
46.2 \\
45.3\end{array}$ & $\begin{array}{c}25 \\
24 \\
22.3\end{array}$ & $\begin{array}{l}96 \\
91 \\
83\end{array}$ \\
\hline 6 & 32 & $\begin{array}{l}\mathrm{T} 0 \\
\mathrm{~T} 1 \\
\mathrm{~T} 2\end{array}$ & $\begin{array}{l}90 \\
85 \\
83\end{array}$ & 173 & $\begin{array}{l}30.0 \\
28.4 \\
27.7\end{array}$ & $\begin{array}{l}85 \\
81 \\
80\end{array}$ & $\begin{array}{l}42.1 \\
36.3 \\
35.6\end{array}$ & $\begin{array}{c}22 \\
19.9 \\
17\end{array}$ & $\begin{array}{c}100 \\
90 \\
88\end{array}$ \\
\hline 7 & 30 & $\begin{array}{l}\text { T0 } \\
\text { T1 } \\
\text { T2 }\end{array}$ & $\begin{array}{c}67 \\
63.8 \\
62\end{array}$ & 157 & $\begin{array}{l}27.1 \\
25.8 \\
25.1\end{array}$ & $\begin{array}{l}74 \\
69 \\
68\end{array}$ & $\begin{array}{l}36.6 \\
33.5 \\
32.4\end{array}$ & $\begin{array}{c}28.6 \\
25 \\
24.2\end{array}$ & $\begin{array}{l}94 \\
91 \\
88\end{array}$ \\
\hline 8 & 25 & $\begin{array}{l}\text { T0 } \\
\text { T1 } \\
\text { T2 }\end{array}$ & $\begin{array}{l}75 \\
70 \\
69\end{array}$ & 158 & $\begin{array}{l}30.0 \\
28.0 \\
27.6\end{array}$ & $\begin{array}{l}83 \\
78 \\
74\end{array}$ & $\begin{array}{l}36.0 \\
34.6 \\
34.0\end{array}$ & $\begin{array}{c}23 \\
21 \\
18.7\end{array}$ & $\begin{array}{c}100 \\
93 \\
87\end{array}$ \\
\hline 9 & 21 & $\begin{array}{l}\text { T0 } \\
\text { T1 } \\
\text { T2 }\end{array}$ & $\begin{array}{l}98 \\
93 \\
90\end{array}$ & 158 & $\begin{array}{l}39.2 \\
37.2 \\
36.0\end{array}$ & $\begin{array}{l}92 \\
85 \\
83\end{array}$ & $\begin{array}{l}50.2 \\
47.9 \\
45.4\end{array}$ & $\begin{array}{c}27.6 \\
26.7 \\
23\end{array}$ & $\begin{array}{l}85 \\
82 \\
79\end{array}$ \\
\hline 10 & 41 & $\begin{array}{l}\text { T0 } \\
\text { T1 } \\
\text { T2 }\end{array}$ & $\begin{array}{c}77 \\
71.5 \\
69\end{array}$ & 152 & $\begin{array}{l}33.3 \\
30.9 \\
29.8\end{array}$ & $\begin{array}{l}84 \\
76 \\
76\end{array}$ & $\begin{array}{l}41.0 \\
38.7 \\
30.1\end{array}$ & $\begin{array}{c}29.1 \\
27 \\
24.4\end{array}$ & $\begin{array}{c}100 \\
91 \\
84\end{array}$ \\
\hline
\end{tabular}


A)

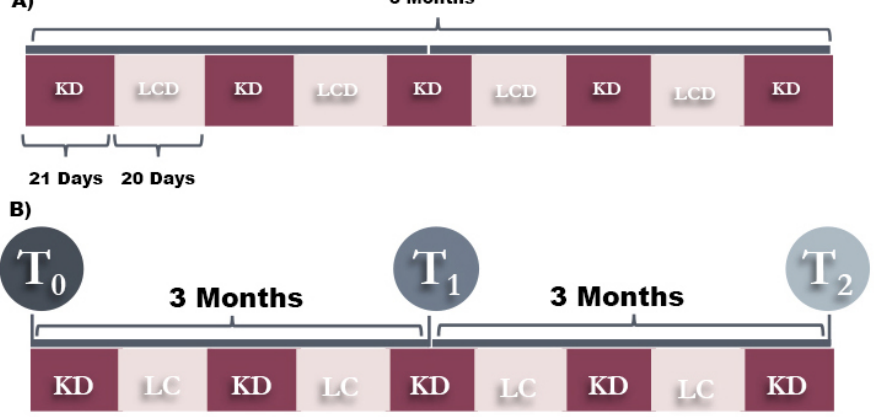

C)

Ketogenic Diet

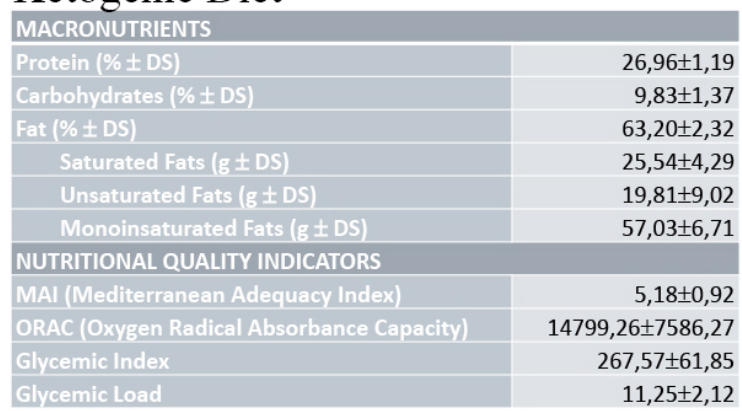

Low Carb Diet

\begin{tabular}{|c|c|}
\hline \multicolumn{2}{|l|}{ MACRONUTRIENTS } \\
\hline Protein (\% $\pm \mathrm{DS})$ & $26,96 \pm 1,19$ \\
\hline Carbohydrates (\% $\%$ DS) & $38,83 \pm 1,37$ \\
\hline Fat $(\% \pm \mathrm{DS})$ & $33,20 \pm 2,32$ \\
\hline Saturated Fats ( $\mathrm{g} \pm \mathrm{DS})$ & $35,54 \pm 4,29$ \\
\hline Unsaturated Fats ( $\mathrm{g} \pm \mathrm{DS}$ ) & $28,81 \pm 9,02$ \\
\hline Monoinsaturated Fats ( $\mathrm{g} \pm \mathrm{DS}$ ) & $37,03 \pm 6,71$ \\
\hline \multicolumn{2}{|l|}{ NUTRITIONAL QUALITY INDICATORS } \\
\hline MAI (Mediterranean Adequacy Index) & $6,18 \pm 0,92$ \\
\hline ORAC (Oxygen Radical Absorbance Capacity) & $15866,36 \pm 7586,27$ \\
\hline Glycemic Index & $323,57 \pm 71,85$ \\
\hline Glycemic Load & $32,25 \pm 2,12$ \\
\hline
\end{tabular}

Figure 1. Cyclic Alternation of Ketogenic (21 days) and Low Carb Diet (20 days) for 6 months protocol (A) and alternation of T0 (time 0$)$, T1 (3 months) and T2 (6 months) period of cyclic diet (B). The table show the different amount of macronutrients and nutritional indicators, that are present in our Ketogenic and Low Carb diet (C)

this basis, we can declare that only 3 of them have not adhered to the proposed diet and have been excluded from the study.

\section{Dietary treatment}

We selected 2 different diet, ketogenic (KD) and very low carb (VLCD), in which the daily kcal amount was calculated subtracting to the estimated basal metabolism $1000 \mathrm{kcal} /$ day. We considered a diet as ketogenic, when a number of carbohydrates were $<20 \mathrm{~g} /$ day. The KD aimed at a daily energy intake of $750-800 \mathrm{kcal}$ per day, with $35-40 \%$ of calories from proteins (corresponding to $1.2 \mathrm{~g} / \mathrm{kg}$ of ideal body weight), $45-50 \%$ from fat ( $<10 \%$ of calories from saturated fat), and $10 \%$ from carbohydrates $(<20 \mathrm{~g})$. KD provided an intake of $20 \mathrm{mg}$ of fiber per day. The half of the amount of daily protein was reached using food supplementation, contained: whey protein (15 g/portion), carbohydrate ( $8 \mathrm{~g} /$ portion), fat ( $20 \mathrm{~g} /$ portion), isoleucine ( $2 \mathrm{~g} /$ portion), ornithine alpha-ketoglutarate ( $1 \mathrm{~g} /$ portion), L-citrulline (1.5g/portion), L-tryptophan (0.5 g/portion), potassium citrate (1 g/portion), for a total of $64 \mathrm{kCal}(268 \mathrm{KJ})$ (Cyclicity Diet ${ }^{\oplus}$, Manoppello, Italy).
The VLCD aimed at daily energy intake of 950-1150 kcal with 20 $30 \%$ of calories from proteins (corresponding to $0.9 \mathrm{~g} / \mathrm{kg}$ of ideal body weight), $45-50 \%$ from fat ( $<10 \%$ of calories from saturated fat) and $20-25 \%$ of calories from carbohydrates $(<50 \mathrm{~g} ;>80 \%$ from simple sugars). Both treatments provided an intake of $20 \mathrm{~g}$ of fiber per day. In all protocols, a multivitamin, proper integration of mineral salts and an alkalizing product were prescribed, when they were not included in the products. The correct administration of diet was evaluated by urinary keto-stick every 3 days of diet to follow the compliance of women to protocol.

\section{Anthropometric measurements}

At each evaluation, after a 12-hour overnight fast, all subjects underwent anthropometric measurements. All the individuals were instructed to take off their clothes and shoes before undergoing the measurements. Waist and hip circumferences were taken using a flexible steel metric tape to the nearest $0.5 \mathrm{~cm}$. Hip circumference was measured according to International Society for the Advancement of Kin anthropometry protocol taken at the greatest posterior protuberance of the buttocks. Waist circumference was measured just above the iliac crest to the nearest $0.1 \mathrm{~kg}$, using a balance scale (Invernizzi, Rome, Italy). Height (m) was measured using a stadiometer to the nearest 0.1 $\mathrm{cm}$ (Invernizzi, Rome, Italy). BMI was calculated using the formula: BMI = body weight $/$ height $2(\mathrm{~kg} / \mathrm{m} 2)$.

\section{Ovarian ultrasound}

At each evaluation, a single experienced investigator performed all the ultrasound scans using a Voluson-S6 (GE Healthcare Ultrasound) and a $5-9-\mathrm{MHz}$ transvaginal volume transducer, which has $3 \mathrm{D}$ ultrasound scanning modes. Antral follicle number was measured using a $3 \mathrm{D}$ ultrasound dataset, with Sonography-based Automated Volume Count (SonoAVC ${ }^{\mathrm{TM}}$, GE Healthcare Ultrasound, Zipf, Austria), as previously described by other authors [13].

The acquired 3D ultrasound datasets were displayed in the multiplanar view. The image were optimized to generate a threedimensional volume of interest (VOI) and to ensure that the whole ovary was included without extra-ovarian information.

SonoAVC was applied for automatically identifying and quantifying hypoechoic areas within a 3D ultrasound dataset. Post-processing, involving the manual identification of follicles not included in the previous automated analysis, was then used to ensure that all antral follicles were counted. The total antral follicle count for each subject was recorded to the nearest millimeter, starting from $2.0 \mathrm{~mm}$ up to a maximum of $10.0 \mathrm{~mm}$ [14].

In anovulatory women, in absence of recruited follicles or corpora lutea, every day was considered reliable to perform the ultrasound. Each woman was asked to annotate her menstrual period in an agenda, in order to evaluate the effect of the dietary regimen on the menstrual cyclicity [15]. Sonographers, did not know the patients and were not informed whether them were under treatment or the kind of possible treatment.

\section{Biochemical analysis}

Blood tests were performed at each time of evaluation, after a 12hour overnight fast. Blood samples $(10 \mathrm{~mL})$ were collected into sterile tubes containing EDTA (Vacutainer ${ }^{\circledR}$ ). All materials were immediately placed on ice and plasma was separated by centrifugation at $1600 \AA ̊$ $\mathrm{g}$ for $10 \mathrm{~min}$ at $4^{\circ} \mathrm{C}$. Laboratory tests included Insulin and glucose and 

patients

subsequently we calculated Homa Index. Insulin clinical analyses were carried out with an ADVIA ${ }^{\star} 1800$ Chemistry System (Siemens Healthcare, Munich, Germany). Plasma glucose concentrations were measured using the glucose oxidase method with an automated glucose analyzer (COBAS INTEGRA 400, Roche Diagnostics, Indianapolis, IN, USA). All tests were performed using the same lot of reagents or assay plates to minimize variability due to differences in reagent lots. Analyses were carried out at the accredited Clinical Chemical Laboratories of the "University Hospital Tor Vergata" of Rome, Italy. For each patient, at each evaluation, HOMA index was used and the cut-off value for insulin resistance was defined by our laboratory in another apparently normal reference population.

\section{Statistical analysis}

Paired t-test or a non-parametric Wilcoxon test were performed to evaluate differences at baseline and after a nutritional intervention. The differences between parameter at baseline and after diet were calculated as the follow:

$$
\Delta \%=[(\mathrm{Z}-\mathrm{W}) / \mathrm{W}] \AA ̊ \Omega 0(9)
$$

where $\Delta \%$ is the percentage variation of each parameter, calculated as the ratio of absolute variation to the base value. The null hypothesis was rejected at the 0.05 level of probability. 3 patients who dropped out were not statistically evaluated after $\mathrm{T} 0$.

\section{Results}

Fifty-seven PCOS women were included in the study. In Figure 2 a decrease in HOMA index, in fat mass and in insulin resistance in women treated with cyclic protocol is showed. Interestingly, we found a similar positive effect both in metabolically unhealthy but normal weight PCOS women and in metabolically unhealthy obese PCOS women treated by our KD and VLCD protocol (Figure 2D). In particular, as indicated in Figure 2A, HOMA index decreased significantly $(\mathrm{p}<0.01)$ in our population at T2 $(-61 \%)$ but not at T1. In the same manner, fat mass measured in $\mathrm{Kg}$, decreased moderately in $\mathrm{T} 2(-30 \%)$ compared with T0 (Figure 2C). Figure 2B shows that 7 of 57 insulin resistant patients enrolled into the study, became insulin sensitive at the end of the 6 months protocol.

Regarding gynecological features, in Figure 3 the recovery in menstrual cycle is showed, correlated to the decrease in ovarian follicle count. In Figure 3A, in particular, a significant improvement in menstrual cycle, respectively in $\mathrm{T} 1(+90 \%)$ and $\mathrm{T} 2(+78 \%)$ compared
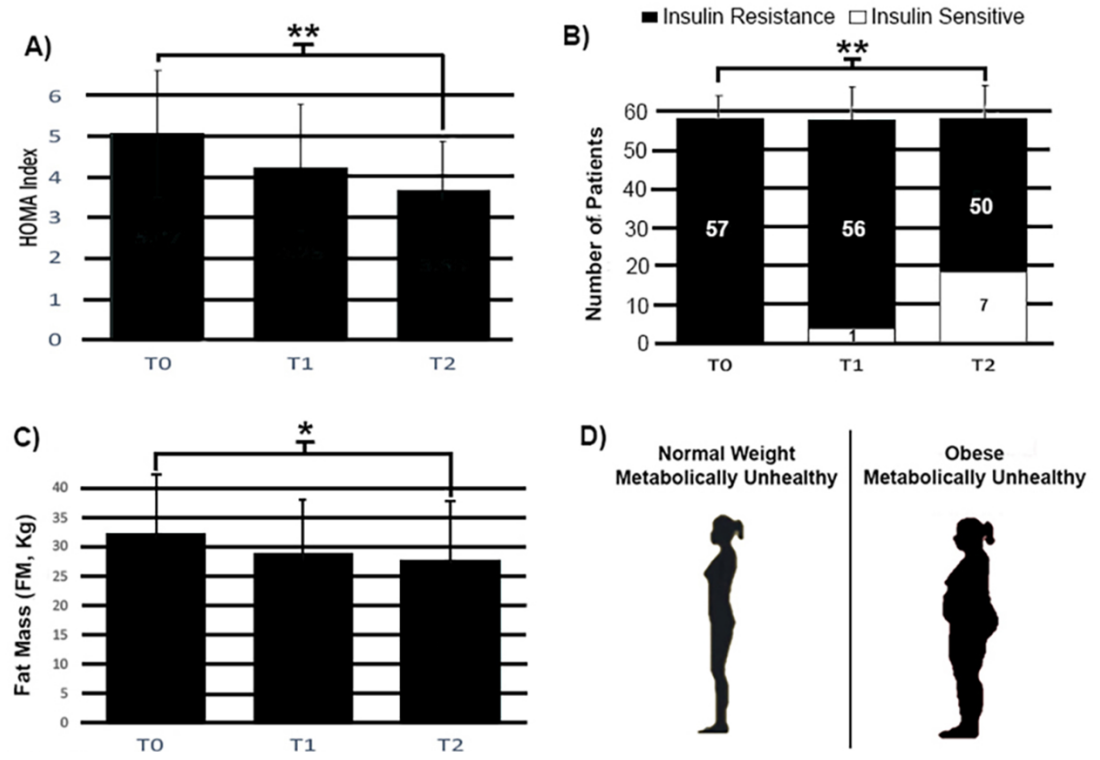

Figure 2. Effects of treatments of Cyclic diet on Homa index (A), fat mass (C) and insulin resistance (B) at T0, T1 and T2. We can see that the women that could have insulin resistance and consequently PCOS, are obese metabolically unhealthy but also normal weight metabolically unhealthy (D). 3 patients who dropped out were not statistically evaluated after T0. Each bar represents $\%$ change ( \pm S.E.M) with respect to women at T0. Percentage changes were determined by a paired t-test or a non-parametric Wilcoxon test

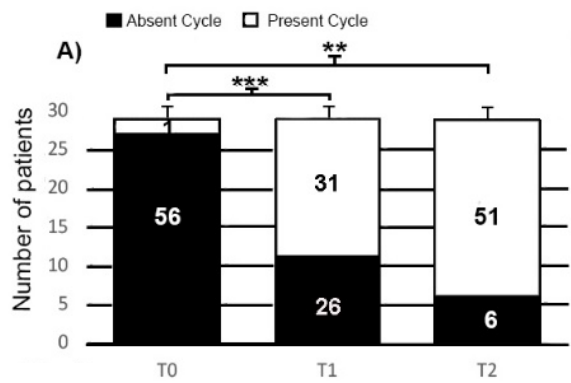

B)

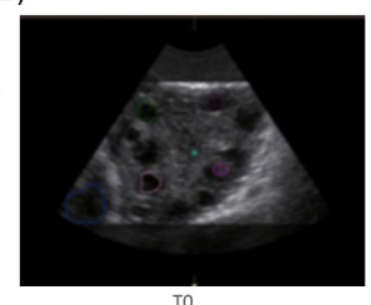

28 Follicles

Ultrasound Ovaries

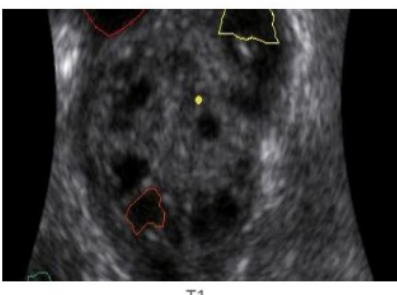

20 Follicles

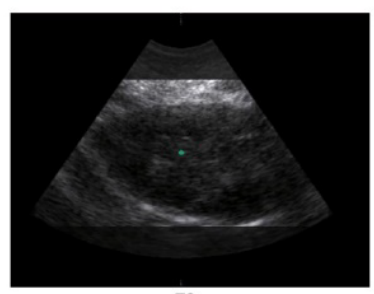

10 Follicles

Figure 3. Effect of Cyclic diet treatment on menstrual cycle recovery at $\mathrm{T} 0, \mathrm{~T} 1$ and $\mathrm{T} 2 \mathrm{(A)}$. The recovery effect is due to the reduction in ovarian antral follicles from 28 (T0) to 10 (T2) measured with ultrasound (B). 3 patients who dropped out were not statistically evaluated after T0 and 1 women on 57 had 3 menstrual cycle per year at T0, whereas, other 56 patients it were in amenorrhea. Each bar represents \% change ( \pm S.E.M) with respect to women at T0. Percentage changes were determined by a paired t-test or a non-parametric Wilcoxon test" 

patients

with T0 is showed, while Figure $3 \mathrm{~B}$ shows the reduction in ovarian follicles, from 28 at $\mathrm{T} 0$ to 20 at $\mathrm{T} 1$ and 10 at $\mathrm{T} 2$ (Figure 3B). 1 women on 57 had 3 menstrual cycle per year at T0, whereas, other 56 patients it were in amenorrhea.

\section{Discussion}

The pathogenesis of PCOS and the clinical factors of the syndrome show a strong relationship with insulin resistance and hyperandrogenism. Consequently, a specific nutritional approach could improve insulin sensitivity and reduce fat mass. Specific cyclic diet can be an efficient method to reduce hyperandrogenism, normalize ovulation, and reduce the signs and symptoms of PCOS with a reduction in cardiovascular and neoplastic risk [16]. Indeed, the guidelines on the treatment of PCOS indicate the change in lifestyle as the first therapeutic intervention; diet and physical activity are effective for weight loss and body composition modification. Recent studies on the treatment of obesity, have highlighted the efficacy of the KD. Therefore, due to the presence of common pathogenic mechanisms, the current guidelines for PCOS will have to be reconsidered. There are no known curative therapies for PCOS, though anti-diabetic medications improve many of the metabolic abnormalities such as insulin resistance [17], elevated serum testosterone, and total cholesterol levels [18]. Recent studies have shown that a low-carbohydrate and KD are linked to weight loss and a decrease in insulin resistance with strong benefits for PCOS patients [12].In accordance with previous acknowledgements, it was shown in the present study that a low-calorie diet therapy with high nutritional index, could be effective in PCOS patients. In particular, the cycle of two different diets, KD and VLCD, showed significant results over a six month treatment period. Carbohydrate intake was lower than $20 \mathrm{~g}$ in $\mathrm{KD}$ and $50 \mathrm{~g}$ in VLCD because, as already demonstrated, the reduction of carbohydrates and calories improves insulin sensitivity. The protein intake in both dietary therapies were personalized [19] in order to save the lean mass for maintaining the results obtained [20]. The lipid supply was particularly rich in mono and poly-unsaturated fatty acids, elements that guaranteed anti-inflammatory and insulin-sensitizing effects [21]. Our findings are similar to a previous clinical series of the use of a low $(100 \mathrm{~g} / \mathrm{d})$ carbohydrate, high saturated fat diet in 15 women with PCOS [22]. However, our new approach using a cycle KD and VLCD diet appears to have a specific effect on compliance and insulin resistance as well as on long term diet with recovery of menstrual cycle. Our hypothesis about the mechanism of antral follicle count reduction and menstrual cycle recovery, is in accordance with other study [23] that explain that the hyperinsulinemia of PCOS appears to increase androgen secretion from the ovary as well as to decrease circulating sex hormone binding globulin (SHBG). Cycle KD and VLCD may lead to a reversal of these processes. Reduction in hyperinsulinemia could be due to the KD through decrease stimulation of ovarian androgen production as well as increase SHBG levels, synergistically limiting the amounts of circulating free-androgens in the serum [24].

The high MAI and ORAC index of diets were representative of a Mediterranean pattern, despite the different distribution of macronutrients. We hypothesize that the clinical and nutritional goals achieved are due to diet therapy, given that no drugs were administered and no sports activities were performed. Significant weight loss was observed $(-5.5 \%)$ in the first two months and reached $-8.66 \%$ at the end of treatment. This weight loss was accompanied by a reduction in fat mass that together with the appearance of menstrual bleeding showed an improvement in metabolic risk parameters. In particular, the glucidic profile has improved in accordance with the initial hypotheses of the study. At least in clinical populations, PCOS is associated with a greater rate of overweight and obesity [25]. However, studies in unselected (medically unbiased) adult populations note that the prevalence of PCOS does not seem to be different between normal-weight and obese women [26] and that the BMI in those women is significantly lower than in the referred population, implying a potential referral bias in this population group [27]. In adults, obesity has been associated with increased insulin resistance, type 2 diabetes, dyslipidemia, and cardiovascular disease risk [28]. A recovery of ovulatory activity and consequently menstrual cycle were highlighted in all patients after three months of cyclic diet therapy. Furthermore, 96.6\% of enrolled women had at least one menstrual cycle in the first trimester. This result was not maintained in the second trimester, in which 6 patients out of 29 (20.7\%) declared absence of menstrual cycle. In one patient with previously irregular menstrual cycle, 10 days after completing the treatment, a pregnancy was diagnosed. Furthermore, at T1 time the ultrasound scans of the patients did not shows significant changes compared to $\mathrm{T} 0$, despite the resumption of the menstrual cycle; but after 6 months a significant reduction in the number of antral follicles was observed in the patients. However, to confirm the preliminary results of this study it is necessary to complete the long-term follow-up in order to verify if the effects obtained are maintained even after some time and after the adoption of a less restrictive, more sustainable eating behavior. A weak point of this study is the lack of a control group.

In conclusion, in this pilot study, low carbohydrates therapy provided positive results in patients affected by obesity and type 2 diabetes, so these dietetic regimens could represent a fascinating dietetic treatment for the management of PCOS. Given the significant health implications related to PCOS, cyclic diet decreases insulin resistance and recovers menstrual cycle in 51 women on 57 in which we saw after 6 months of KD and VLCD, a significant decrease in fat mass amount, in HOMA index, and in particular in the number of ovarian antral follicles on women with amenorrhea (Figure 3). In addition, after 6 months (but not 3 months), our data has shown significant menstrual cycle recovery. This approach could constitute a novel therapeutic nutritional treatment in women with PCOS and amenorrhea consequently to insulin resistance.

\section{Acknowledgements}

We thank the Italian University Research Ministry (MIUR), Region of Calabria (POR, FSE-2007/2013) for the financial support.

\section{References}

1. Pundir J, Charles D, Sabatini L, Hiam D, Jitpiriyaroj S, et al. (2019) Overview of systematic reviews of non-pharmacological interventions in women with polycystic ovary syndrome. Hum Reprod Update 25: 243-256. [Crossref]

2. March WA, Moore VM, Willson KJ, Phillips DI, Norman RJ, et al. (2010) The prevalence of polycystic ovary syndrome in a community sample assessed under contrasting diagnostic criteria. Hum Reprod 25: 544-551. [Crossref]

3. Bozdag G, Mumusoglu S, Zengin D, Karabulut E, Yildiz BO (2016) The prevalence and phenotypic features of polycystic ovary syndrome: a systematic review and metaanalysis. Hum Reprod 31: 2841-2855. [Crossref]

4. Rocca ML, Venturella R, Mocciaro R, Di Cello A, Sacchinelli A, et al. (2015) Polycystic ovary syndrome: chemical pharmacotherapy. Expert Opin Pharmacother 16: 1369-1393. [Crossref]

5. Balen AH, Morley LC, Misso M, Franks S, Legro RS, et al. (2016) The management of anovulatory infertility in women with polycystic ovary syndrome: an analysis of the evidence to support the development of global WHO guidance. Hum Reprod Update 22: 687-708. [Crossref]

6. Balen AH, Conway GS, Kaltsas G, Techatrasak K, Manning PJ, et al. (1995) Polycystic ovary syndrome: the spectrum of the disorder in 1741 patients. Hum Reprod 10: 2107 2111. [Crossref] 

patients

7. Diamanti-Kandarakis E, Economou F, Palimeri S, Christakou C (2010) Metformin in polycystic ovary syndrome. Ann NY Acad Sci 1205: 192-198.

8. Alebić MŠ, Bulum T, Stojanović N, Duvnjak L (2014) Definition of insulin resistance using the homeostasis model assessment (HOMA-IR) in IVF patients diagnosed with polycystic ovary syndrome (PCOS) according to the Rotterdam criteria. Endocrine 47: 625-630.

9. Stepto NK, Cassar S, Joham AE, Hutchison SK, Harrison CL, et al. (2013) Women with polycystic ovary syndrome have intrinsic insulin resistance on euglycaemichyperinsulaemic clamp. Hum Reprod 28: 777-784. [Crossref]

10. Moran LJ, Hutchison SK, Norman RJ, Teede HJ (2011) Lifestyle changes in women with polycystic ovary syndrome. Cochrane Database Syst Rev 7: CD007506. [Crossref]

11. Yancy WS, Olsen MK, Guyton JR, Bakst RP, Westman EC (2004) A low carbohydrate ketogenic diet versus a low-fat diet to treat obesity and hyperlipidemia. Ann Intern Med 140: 769-777. [Crossref]

12. Boden G, Sargrad K, Homko C, Mozzoli M, Stein TP (2005) Effect of a low carbohydrate diet on appetite, blood glucose levels, and insulin resistance in obese patients with type 2 diabetes. Ann Intern Med 142: 403-411. [Crossref]

13. Balen AH, Laven JS, Tan SL (2003) Ultrasound assessment of the polycystic ovary: international consensus definitions. Hum Reprod Update 9: 505-514. [Crossref]

14. The Rotterdam ESHRE/ASRM-Sponsored PCOS Consensus Workshop Group (2004) Revised 2003 consensus on diagnostic criteria and long-term health risks related to polycystic ovary syndrome. Fertil Steril 81: 19-25. [Crossref]

15. Chun S (2014) Serum luteinizing hormone level and luteinizing hormone/ folliclestimulating hormone ratio but not serum anti-Mullerian hormone level is related to ovarian volume in Korean women with polycystic ovary syndrome. Clin Exp Reprod Med 41: 86-91. [Crossref]

16. Dumesic DA, Lobo RA (2013) Cancer risk and PCOS. Steroids 78: 782-785. [Crossref]

17. Glueck CJ, Moreira A, Goldenberg N, Sieve L, Wang P (2003) Pioglitazone and metformin in obese women with PCOS not optimally responsive to metformin. Hum Reprod 18: 1618-1625.

18. Nestler JE, Jakubowicz DJ, Reamer P, Gunn RD, Allan G (1999) Ovulatory and metabolic effects of d-chiro-inositol in the polycystic ovary syndrome. $N$ Engl $J$ Med 340: 1314-1320. [Crossref]
19. Colica C, Merra G, Gasbarrini A, De Lorenzo A, Cioccoloni G, et al. (2017) Efficacy and safety of very-low-calorie ketogenic diet: a double blind randomized crossover study. Eur Rev Med Pharmacol Sci 21: 2274-2289. [Crossref]

20. Marchetti M, Gualtieri P, Romano L, Merra G (2019) What is the importance of saving lean mass in the treatment of obesity and related diseases? Eur Rev Med Pharmacol Sci 23: 431-432. [Crossref]

21. Colica C, Di Renzo L, Trombetta D, Smeriglio A, Bernardini S, et al. (2017) Antioxidan effects of a hydroxytyrosol-based pharmaceutical formulation on body composition, metabolic state, and gene expression: a randomized double-blinded, placebo-controlled crossover trial. Oxid Med Cell Longev 2017: 2473495. [Crossref]

22. Hays JH, Disabatino A, Gorman RT, Vincent S, Stillabower ME (2003) Effect of a high saturated fat and no-starch diet on serum lipid subfractions in patients with documented atherosclerotic cardiovascular disease. Mayo Clin Proc 78: 1331-1336. [Crossref]

23. Mavropoulos JC, Yancy WS, Hepburn J, Westman EC (2005) The effects of a lowcarbohydrate, ketogenic diet on the polycystic ovary syndrome: A pilot study. Nutr Metab 2: 35. [Crossref]

24. Nestler JE, Powers LP, Matt DW, Steingold KA, Plymate SR, et al. (1991) A direct effect of hyperinsulinemia on serum sex-hormone-binding globulin levels in obese women with the polycystic ovary syndrome. J Clin Endocrinol Metab 72: 83-89.

25. Lim SS, Davies MJ, Norman RJ, Moran LJ (2012) Overweight, obesity and central obesity in women with polycystic ovary syndrome: a systematic review and metaanalysis. Hum Reprod Update 18: 618-637. [Crossref]

26. Yildiz BO, Knochenhauer ES, Azziz R (2008) Impact of obesity on the risk for polycystic ovary syndrome. J Clin Endocrinol Metab 93: 162-168. [Crossref]

27. Ezeh U, Yildiz BO, Azziz R (2013) Referral bias in defining the phenotype and prevalence of obesity in polycystic ovary syndrome. J Clin Endocrinol Metab 98 E1088-E1096. [Crossref]

28. Morgan CL, Jenkins-Jones S, Currie CJ, Rees DA (2012) Evaluation of adverse outcome in young women with polycystic ovary syndrome versus matched, reference controls: a retrospective, observational study. J Clin Endocrinol Metab 97: 3251-3260. [Crossref]

Copyright: (C2020 Avolio E. This is an open-access article distributed under the terms of the Creative Commons Attribution License, which permits unrestricted use, distribution, and reproduction in any medium, provided the original author and source are credited. 